\title{
Dissoziationsbedingte serologische Kreuzreaktionen bei
} Schweine-Enterotoxämie-Coli-Typen

\begin{tabular}{|l|l|l}
\hline H.U. & Bertschinger \\
\hline
\end{tabular}

Veterinär-Bakteriologisches Institut der Universität Zürich

Adresse des Autors: Dr. H. U. Bertschinger, Veterinär-Bakteriologisches Institut der Universität Zürich, WinterthurerStrasse 270, 8057 Zürich (Schweiz)

Bei E. coli kommt es zu einer besonderen Art der Dissoziation, die wir bisher nur bei

Enterotoxämie-Typen beobachten konnten. Die dissoziier-ten Kolonien sind in der Beleuchtung nach Henry von der Ausgangsform unterscheidbar. Sie besitzen ein Antigen, das provisorisch mit X bezeich-net wurde und das beim Kaninchen sehr hohe Agglutinintiter erzeugt. Das XAntigen weist viel Ähnlichkeit mit dem K-Antigen auf. Die O-, B- und L-Antigene werden von der Dissoziation nicht betroffen. Während $\mathrm{X}+-$ Kolonien bei frisch isolierten Feldstämmen nie gefunden wurden, waren sie nach Aufbewahrung bei einem grossen Teil der Stämme mit dem Antigen K 88 (L) nachzuweisen. Durch die regelmässige Prüfung der Kolonie-morphologie lassen sich Schwierigkeiten bei der serologischen Typisierung vermeiden. 